\title{
Original
}

\section{Riñones parejos en trasplante}

\author{
Juan C. Regueiro López, Manuel Leva Vallejo, Rafael Prieto Castro, Francisco Anglada Curado, \\ Francisco Vela Jiménez, Jesús Ruiz García
}

Servicio de Urología. Hospital Regional Universitario Reina Sofía.Córdoba, España

\section{Resumen}

Objetivos: Muchos factores afectan la supervivencia del injerto y paciente en el resultado de un trasplante renal. Estos factores dependen tanto del receptor como del donante. Realizamos un estudio intentando obviar factores que dependen del donante. Revisamos los riñones parejas provenientes de un mismo donante cadáver.

Pacientes y métodos: Analizamos los factores de riesgo en la evolución y seguimiento de 556 trasplantes renales que corresponden a 278 parejas de riñones. Describimos sus diferencias, su significación, la supervivencia de injerto y paciente, su funcionalidad a 3 y 5 años y los factores de riesgo implicados en su función. Se estudian variables inmunológicas y no inmunológicas intentando explicar los resultados inferiores en los injertos que se implantan en segundo lugar. Se reagrupan los riñones parejos en aquellos que no mostraron función pareja inicial dentro de la misma pareja.

Resultados: Los resultados arrojan un discreto empeoramiento en la supervivencia de injerto y paciente para el grupo de segundo implante, cifras superiores de creatinina, sin obtener significación estadística. El estudio por regresión de Cox solo establece como factores de riesgo el rechazo precoz (inferior a tres meses) y la tasa de incompatibilidad DR.

Conclusiones: Este modelo de riñones parejos podría acercarnos de forma más adecuada al análisis de los factores de riesgo en trasplante de donante cadáver, si más pacientes se analizan de la misma forma. Los riñones parejas provenientes del mismo donante no muestran igual función a pesar de compartir las mismas condiciones del donante y del manejo peroperatorio.

Palabras clave: Trasplante renal. Riñones parejas. Supervivencia de injerto. Supervivencia de paciente.

\section{Paired kidneys in transplant}

\section{Abstract}

Objectives: Many factors affect the graft and patient survival on the renal transplant outcome. These factors depend so much of the recipient and donor. We accomplished a study trying to circumvent factors that depend on the donor. We checked the paired kidneys originating of a same donor cadaver.

Patients and method: We examined the risk factors in the evolution and follow-up in 278 couples of kidney transplant. We describe their differences, significance, the graft and patient survival, their functionality in 3 and 5 years and the risk factors implicated in their function. We study immunogenic and no immunogenic variables, trying to explain the inferior results in the grafts that are established secondly. We regroup the paired kidneys in those that they did not show paired initial function within the same couple.

Results: The results yield a discreet deterioration in the graft and patient survival for second group establish, superior creatinina concentration, without obtaining statistical significance. The Cox regression study establishes the early rejection (inferior to three months) and DR incompatibility values like risk factors.

Conclusions: This model of paired kidneys would be able to get close to best-suited form for risk factors analysis in kidney transplant from cadaver donors, if more patients examine themselves in the same way. The paired kidneys originating from the same donor do not show the same function in spite of sharing the same conditions of the donor and perioperative management.

Keywords: Kidney transplant. Paired Kidney. Graft survival. Patient survival.

$\mathrm{M}$ uchos factores afectan la supervivencia del injerto y paciente en el trasplante renal (edad de donante o receptor, sexo, raza, enfermedad primaria de fracaso renal, tiempo de isquemia fría, compatibilidad HLA, transfusión previa, grupos sanguíneos, métodos de preservación, etc.) ${ }^{1-3}$.
Numerosos trabajos demuestran el efecto perjudicial de la "función retrasada inicial" (FRI) o "necrosis tubular aguda" (NTA) sobre la supervivencia del injerto renal $^{4-7}$. La función renal a largo plazo dependerá de numerosos factores provenientes tanto del donante como del injerto. Un tiempo de 
preservación alargado contribuye a la $\mathrm{FRI}^{8-10}$, por ello el ejemplo del donante vivo con mínimo tiempo de isquemia confirma la anterior idea. Largos periodos de preservación contribuyen a instaurar un daño inflamatorio y de reperfusión que desembocan en una FRI y con ello a una peor supervivencia ${ }^{11,12}$. Podemos pensar que los riñones parejas, provenientes del mismo donante nos pueden servir de modelo de análisis más eficaz: extracción y manejo peroperatorio similar ${ }^{13,14}$, preservación en las mismas condiciones, trasplantados por el mismo grupo quirúrgico con misma técnica quirúrgica y con igual seguimiento posoperatorio inmediato. Por ello intentamos en esta revisión, minimizar los factores dependientes del donante y periextracción. Analizamos los factores de riesgo en la evolución así como los resultados de 556 trasplantes renales procedentes de cadáver, realizados durante el periodo 1985-2008, revisando 278 parejas de riñones, comparando los dos grupos de receptores que recibieron riñones del mismo donante.

\section{MATERIAL Y MÉTODOS}

Desde 1980 a 2008, se han realizado 1133 trasplantes renales en nuestro centro. Para los propósitos de esta revisión, solo estudiaremos los riñones provenientes del mismo donante. Incluimos 556 trasplantes de 278 donantes. Suelen ser realizados de forma consecutiva por el mismo grupo quirúrgico. Los 278 se agruparon en grupo 1 (primer implante de riñón del mismo donante) y grupo 2 (segundo implante de riñón del mismo donante).

La edad media del donante fue 44,66 años (rango 7-83). 62\% fueron varones, y la causa del fallecimiento fue accidente hemorrágico en el 70\% y traumatismo craneal en el 30\%. Fueron extracciones multiorgánicas en un $72 \%$.

Todos los receptores se encontraban en diálisis previa, habiendo recibido un número conocido de transfusiones durante el tiempo de lista de espera. La histocompatibilidad era conocida en todos y su crossmatch negativo necesario. La compatibilidad de edad es un criterio de selección. Hemodiálisis se realizo en las 24 horas previas al trasplante. Las pautas de inmunosupresión han sido variadas.

Tabla 1
Consideramos FRI donde fue necesaria una sesión de hemodiálisis en la primera semana o un mantenimiento de los niveles de creatinina superiores a $2 \mathrm{mg} / \mathrm{dl}$ en la primera semana ${ }^{15}$.

En los episodios de rechazo agudo se utilizó como terapia inicial bolos de metilprednisolona, con posterior uso de anticuerpos poli o monoclonales en caso de no respuesta inicial a corticoides. Consideramos rechazo precoz el que se produce en los seis primeros meses de seguimiento.

Se define pérdida de injerto cuando el paciente vuelve a diálisis, cuando se realiza la trasplantectomía o el fallecimiento del paciente con riñón funcionante.

El estudio estadístico se basa en comparación de frecuencias de variables cuantitativas mediante la prueba $\mathrm{T}$ de variables independientes, tablas de contingencia con Chi-2 para cualitativas, estudio de supervivencia tipo Kaplan Meier (con comparativa de Long-Rank) y la regresión logística tipo Cox para valoración de riesgo (SPSS 15,01).

\section{RESULTADOS}

Los 556 trasplantes se dividieron en grupo 1 y 2 de acuerdo al orden de cirugía con respecto a la otra cirugía del mismo donante. Las características de los IMPLANTES de estos dos grupos se reflejan en la Tabla 1. Los resultados funcionales se expresan en la Tabla 2.

\begin{tabular}{lccc}
\hline & Grupo 1 & Grupo 2 & Valor de p \\
\hline Edad media del receptor & 45,07 & 45,97 & NS \\
Edad media del donante & 44,73 & 44,60 & NS \\
Razón receptor hombre/mujer & $184 / 94$ & $176 / 102$ & NS \\
Días de ingreso & 25,04 & 21,15 & $\mathrm{P}<0,05$ \\
Trasfusiones previas & 3,51 & 3,94 & NS \\
Máxima PRA (\%) & 17,79 & 20,23 & NS \\
Tiempo en lista de espera (meses) & 46,10 & 59,11 & $\mathrm{P}<0,05$ \\
Incompatibilidad DR & 0,77 & 0,78 & $\mathrm{NS}$ \\
Incompatibilidad A & 1,09 & 1,06 & $\mathrm{NS}$ \\
Incompatibilidad B & 1,21 & 1,26 & $\mathrm{NS}$ \\
Tiempo de isquemia fría (h) & 16,28 & 20,74 & $\mathrm{P}=0,09$ \\
FRI (Núm.-\%) & $53(19,06)$ & $58(20,86)$ & $\mathrm{NS}$ \\
Rechazo precoz & 0,12 & 0,16 & $\mathrm{P}<0,05$ \\
Rechazo total & 0,18 & 0,22 & $\mathrm{P}<0,05$ \\
\hline
\end{tabular}


Los dos grupos son muy similares, a excepción del tiempo de lista de espera $(p<0,05)$ que fue significativamente mayor en el grupo 2 , y los tiempos de isquemia fría que fueron obligadamente más cortos en el grupo 1 (4 horas y media). Los tiempos de seguimiento medios han sido respectivamente 5,97 y 5,91 años.

En lo referente al éxito del trasplante a corto o medio plazo, encontramos mayor número de FRI en el grupo 2, aunque no significativo. Los valores de creatinina fueron diferentes a los tres meses del implante, modificándose y unificándose estos valores en los controles posteriores a largo plazo. Las supervivencias de injerto y paciente muestran una ligera tendencia a cifras superiores en el grupo 1 a los tres años, equilibrándose posteriormente.

Aunque los niveles de incompatibilidades HLA son semejantes en ambos grupos, existe una cierta tendencia no estadística a una mayor tasa de P.R.A. (panel reactivo de anticuerpos) en el grupo 2 que conlleva un mayor número de episodios de rechazo agudo precoz y total en el grupo 2 .

De las 278 parejas iniciales, 71 parejas mostraron discordante función inicial $(25,5 \%)$. Los datos obtenidos de este subgrupo se muestran en las Tablas 3 y 4.

Comparando los dos grupos de injertos renales principales en lo referente a supervivencia de injerto y de paciente no se encuentran diferencias significativas (Figs. 1 y 2. Log Rank -Mantel-Cox NS).

Comparando el grupo de 71 parejas de injertos renales con funciones iniciales no parejas, tampoco encontramos diferencias en sus tasas de supervivencia de paciente e injerto (Figs. 3 y 4. Long Rank Mantel-Cox NS).

El análisis de regresión logística solo pone de manifiesto dos factores de riesgo como variables independientes: rechazo precoz (RR: 3,6) e incompa-

Tabla 2

Tabla 3

\section{Tabla 4}

\begin{tabular}{lccc}
\hline & Grupo 1 & Grupo 2 & Valor de p \\
\hline Supervivencia trasplante 3 años & 83,09 & 81,29 & NS \\
Supervivencia paciente 3 años & 92,08 & 91,36 & NS \\
Supervivencia trasplante 5 años & 65,10 & 64,74 & NS \\
Supervivencia paciente 5 años & 76,97 & 77,69 & NS \\
Creatinina 3 meses (mg/dl) & 1,6 & 2,07 & P<0,05 \\
Creatinina 6 meses (mg/dl) & 2,20 & 2,26 & NS \\
Creatinina 12 meses (mg/dl) & 1,65 & 1,68 & NS \\
Creatinina 3 años & 1,75 & 1,80 & NS \\
Creatinina 5 años & 1,68 & 1,80 & NS \\
Creatinina 10 años & 1,70 & 1,68 & NS \\
\hline
\end{tabular}

\begin{tabular}{lccc}
\hline & Grupo 1 & Grupo 2 & Valor de p \\
\hline Edad media del receptor & 47,04 & 47,18 & $\mathrm{NS}$ \\
Edad media del donante & 48,99 & 50,43 & $\mathrm{NS}$ \\
Días de ingreso & 31,21 & 28,04 & $\mathrm{NS}$ \\
Trasfusiones previas & 2,99 & 3,79 & $\mathrm{P}=0,059$ \\
Máxima PRA (\%) & 23,11 & 27,56 & $\mathrm{NS}$ \\
Tiempo en lista de espera (meses) & 55,8 & 69,5 & $\mathrm{NS}$ \\
Incompatibilidad DR & 0,77 & 0,92 & $\mathrm{NS}$ \\
Incompatibilidad A & 1,07 & 1,05 & $\mathrm{NS}$ \\
Incompatibilidad B & 1,17 & 1,18 & $\mathrm{NS}$ \\
Tiempo de isquemia fría (h) & 15,64 & 19,66 & $\mathrm{P}<0,05$ \\
Rechazo precoz & 0,17 & 0,20 & $\mathrm{NS}$ \\
Rechazo total & 0,23 & 0,23 & $\mathrm{NS}$ \\
\hline
\end{tabular}

\begin{tabular}{lccc}
\hline & Grupo $\mathbf{1}$ & Grupo 2 & Valor de p \\
\hline Supervivencia trasplante 3 años & 80,28 & 78,87 & NS \\
Supervivencia paciente 3 años & 95,77 & 91,54 & NS \\
Supervivencia trasplante 5 años & 59,15 & 54,92 & NS \\
Supervivencia paciente 5 años & 78,87 & 76,74 & NS \\
Creatinina 3 meses (mg/dl) & 2,26 & 2,43 & NS \\
Creatinina 6 meses (mg/dl) & 2,52 & 2,53 & NS \\
Creatinina 12 meses (mg/dl) & 1,77 & 1,80 & NS \\
Creatinina 3 años & 1,81 & 1,77 & NS \\
Creatinina 5 años & 1,68 & 1,79 & NS \\
Creatinina 10 años & 1,45 & 1,74 & NS \\
\hline
\end{tabular}

tiblidad DR (RR: 1,9) El resto de variables estudiadas no muestra significación al intentar encontrar factores de riesgo en la función del injerto.

\section{DISCUSIÓN}

El fin de este estudio fue investigar los factores que pueden influir en la supervivencia del injerto 


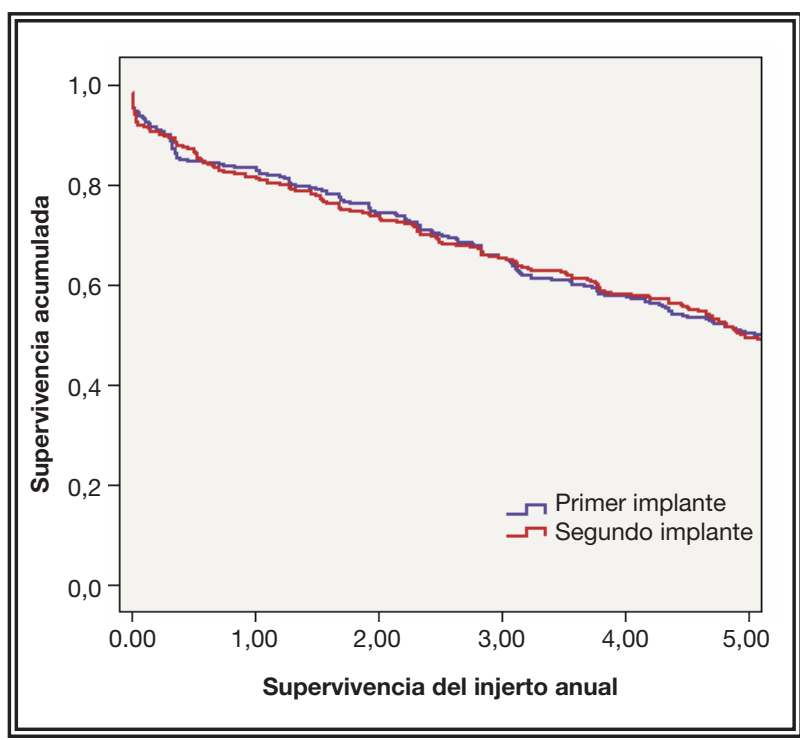

\section{FIGURA 1}

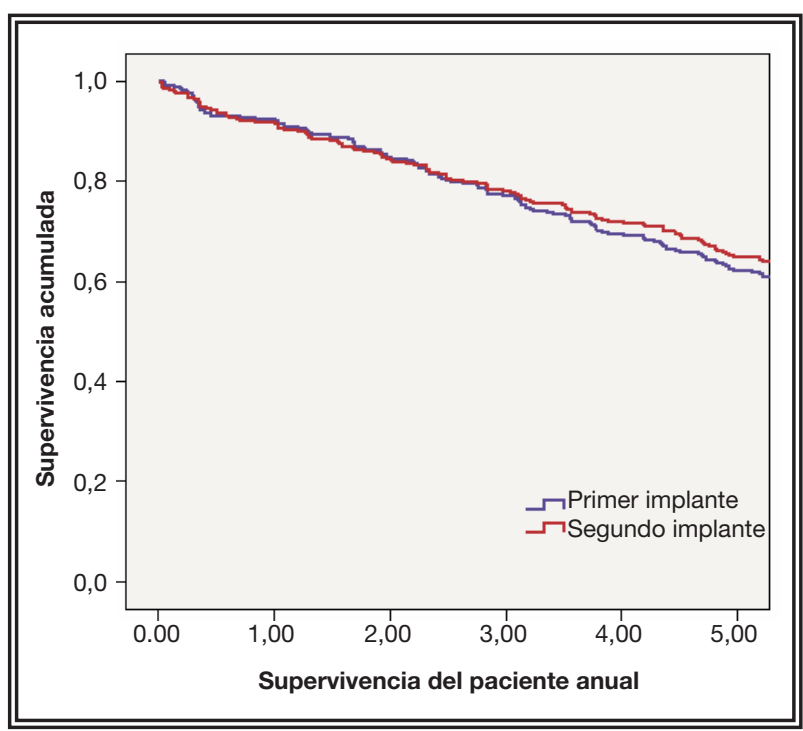

FIGURA 2

minimizando el impacto de los factores de donante al examinar riñones parejos. Se conoce la asociación de mayor tiempo de isquemia con la $\mathrm{FRI}^{9,16}$. EL grupo 2 (segundo implante) de riñones parejos presentan un tiempo de isquemia superior medio de 4.5 horas con respecto al grupo 1 (primer implante), lo cual puede explicar la tasa más alta de FRI en el grupo $2(20,86 \%)$ en relación al grupo $1(19,06 \%)$. Las tasas de supervivencia de injerto a los 3 y 5 años, son superiores para el grupo 1, sin llegar a ser significativas. El tiempo de espera es superior para el grupo 2, y esto podría explicar los datos de las diferencias observadas en la supervivencia del paciente ${ }^{17}$.

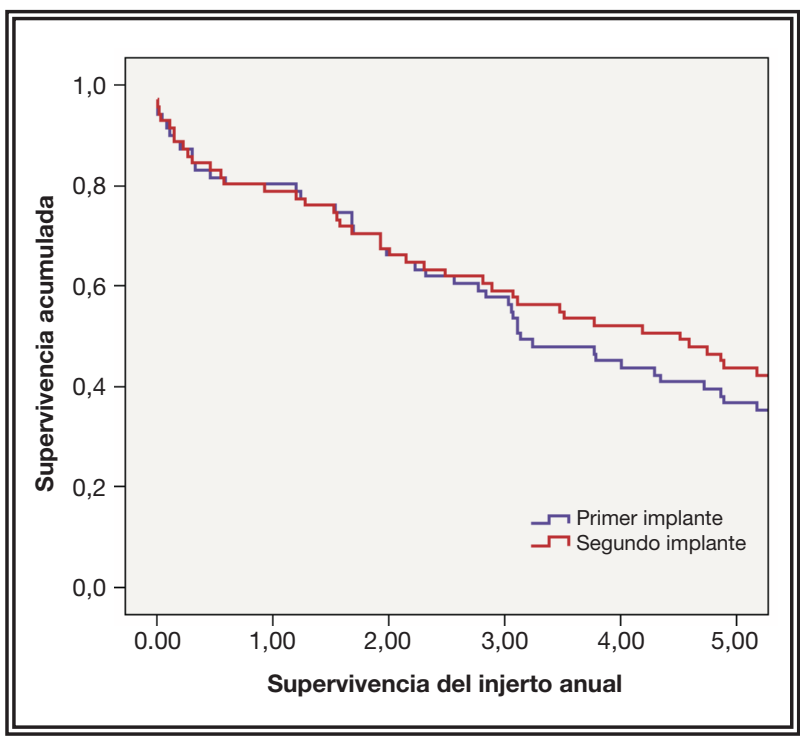

\section{FIGURA 3}

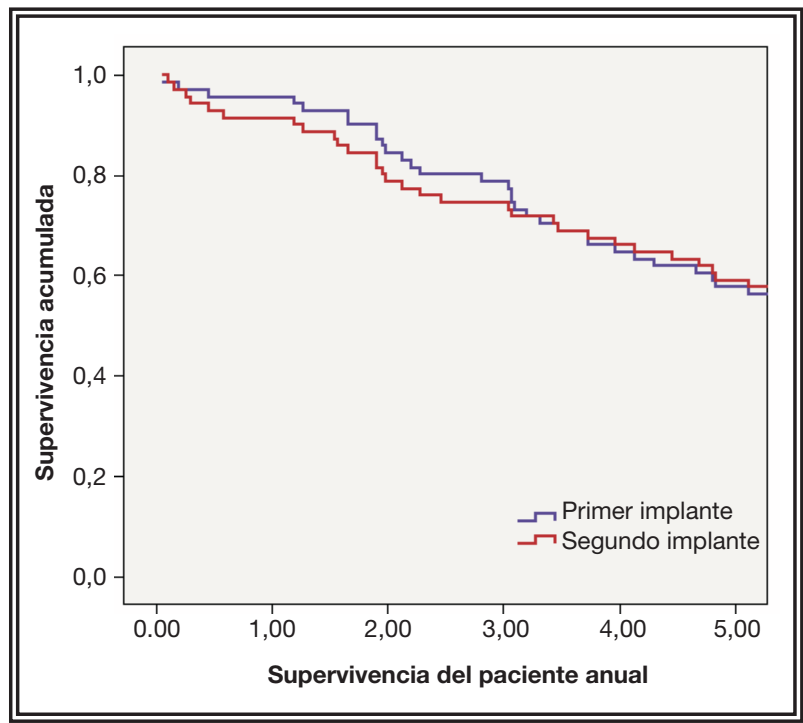

FIGURA 4

Los episodios de rechazo agudo precoz (inferior a 6 meses) y el total de episodios de rechazo agudo son superiores en el grupo 2, lo cual también explicaría las diferencias observadas en la supervivencia de injerto.

Aunque los injertos del grupo 1 mantienen una funcionalidad inicial mejor que el grupo 2 (cifras de creatinina a tres meses: 1,6 vs 2,07 ), esto se equilibra con el paso del tiempo.

Intentando aproximarnos mas a los factores que pueden influir en la supervivencia final del injerto, recogimos los datos de los riñones parejos, que no habían presentado una función pareja, es decir, provienen del mismo donante pero uno de ellos pre- 
senta función inicial inmediata y la otra pareja presenta una FRI. En estos casos solo encontramos como causante de esta diferencia, los tiempos diferentes de isquemia fría (4 horas). Sin embargo, al igual que el grupo total, las diferencias en supervivencia de injerto y de paciente beneficiaban al grupo 1 pero no arrojaban significación estadística. El aspecto funcional del injerto muestra semejanzas a las curvas de supervivencia, con mejores cifras generales de creatinina en el grupo 1, sin ser significativas clínicamente.

Todas las variables son discretamente superiores en el grupo 2 (histocompatibilidad, tiempo de espera, tasa de panel de anticuerpos, tasa de rechazo agudo) lo cual podría explicar las discretas diferencias observadas en los porcentajes de supervivencia de injerto.

En un análisis convencional, las diferencias de isquemia fría podrían suponer un factor de riesgo para la supervivencia del injerto. Sin embargo el estudio de regresión solo muestra como factores de riesgo de importancia, los aspectos inmunológicos (rechazo precoz e incompatibilidad DR), quedando el resto de variables sin valor de riesgo.

Si comparamos la supervivencia del injerto entre aquellos que presentaron función inicial y los que presentaron FRI, estratificándolos en los grupos 1 y 2 de tiempo de implante, se obtienen diferencias significativas en ambos casos (Long-Rank < 0.005), obteniéndose menor supervivencia de injerto en los que presentaron FRI (Figs. 5 y 6).

Este modelo de riñones parejos podría acercarnos de forma más adecuada al análisis de los factores de riesgo en trasplante de donante cadáver, si más pacientes se analizan de la misma forma.

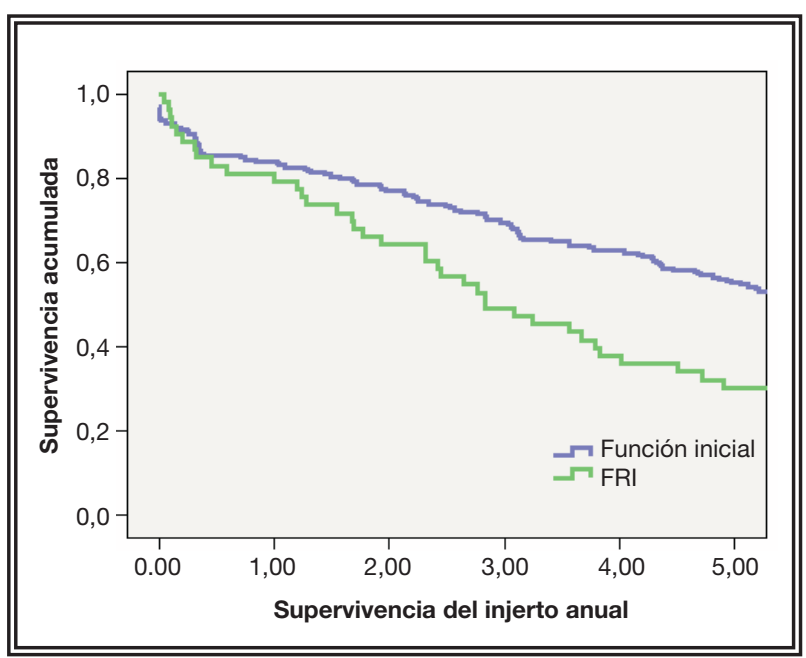

FIGURA 5. Primer implante (riñones con función inicial vs FRI).

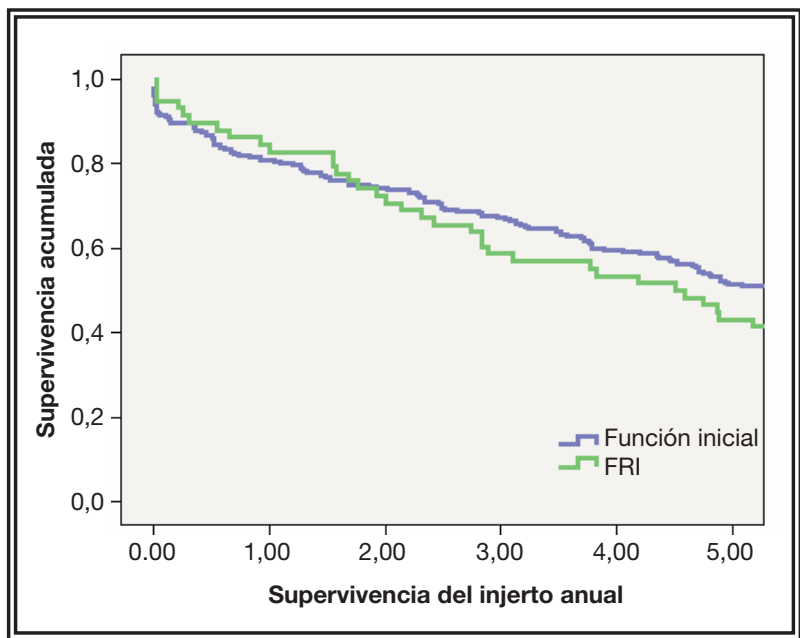

FIGURA 6. Segundo implante (riñones con función inicial us FRI).

Los riñones parejas provenientes del mismo donante no muestran igual función a pesar de compartir las mismas condiciones del donante y del manejo peroperatorio. Sin embargo, tenemos que concluir que las diferencias observadas, fundamentadas en el tiempo de diferencia de implante, no afectan los resultados a largo plazo en supervivencia de injerto y paciente. Otros factores, no analizados en este trabajo (factores del receptor) tienen una alta importancia en el resultado del trasplante, incluyendo el tipo de diálisis ${ }^{12}$.

\section{CONCLUSIONES}

- Pequeños incrementos de isquemia fría contribuyen a una tasa más alta de FRI, sin embargo las supervivencias no llegan a tener significado estadístico. Los tiempos de espera mas alargados inciden en la supervivencia del paciente. El factor inmunológico representado por la incompatibilidad HLA (fundamentalmente DR) y tasa de rechazo agudo temprano colaboran en una mejor supervivencia del injerto precoz.

- La funcionalidad del injerto (cifras de creatinina) del grupo de implante precoz tiende a ser mejor inicialmente, aunque posteriormente se homogeniza con el paso del tiempo.

- Estratificando para ambos grupos la presencia o no de FRI, la supervivencia del injerto dependerá de la función inicial del injerto, arrojando diferencias tanto en el grupo de implante precoz como tardío.

- Este modelo estudio de riñones parejos, nos podría ofrecer datos importantes de factores que influyen en el resultado del trasplante, obviando los factores provenientes de donante cadáver. 


\section{REFERENCIAS}

1. Light JA, Gage F, Kowalski AE, Sasaki TM, Callender CO. Immediate function and cost comparison between static and pulsatile preservation in kidney recipients. Clin Transplant. 1996; 10(3):233-236.

2. Butkus DE, Meydrech EF, Raju SS. Racial differences in the survival of cadaveric renal allografts. Overriding effects of HLA matching and socioeconomic factors. N Engl J Med. 1992; 327(12):840-845.

3. Cho YW, Cecka JM. Cadaver-donor renal retransplants. Clin Transpl. 1993:469-484.

4. Schneider A, Toledo-Pereyra LH, Zeichner WD, Allaben R, Whitten J. Effect of dopamine and pitressin on kidneys procured and harvested for transplantation. Transplantation. 1983; 36(1):110-111.

5. Terasaki PI, Cecka JM, Gjertson DW, Cho Y, Takemoto S, Cohn M. A ten-year prediction for kidney transplant survival. lin Transpl. 1992:501-512.

6. Jang HJ, Kim SC, Kim SK, Han DJ. Paired kidneys as a study model for the analysis of factors affecting cadaveric renal allograft survival. Transplant Proc. 1998; 30(7):3669-3670.

7. Marcén R, Orofino L, Pascual J, de la Cal MA, Teruel JL, Villafruela JJ, et al. Delayed graft function does not reduce the survival of renal transplant allografts.Transplantation. 1998 66(4):461-466.

8. Moreso F, Serón D, Gil-Vernet S, Riera L, Fulladosa X, Ramos $\mathrm{R}$, et al. Donor age and delayed graft function as predictors of renal allograft survival in rejection-free patients. Nephrol Dial Transplant. 1999; 14(4):930-935.

9. Najarian JS, Gillingham KJ, Sutherland DE, Reinsmoen NL, Payne WD, Matas AJ. The impact of the quality of initial graft function on cadaver kidney transplants. Transplantation. 1994; 57(6):812-816.

10. Shoskes DA, Cecka JM. Deleterious effects of delayed graft function in cadaveric renal transplant recipients independent of acute rejection. Transplantation. 1998; 66(12):1697-1701.
11. Koo DD, Welsh KI, Roake JA, Morris PJ, Fuggle SV. Ischemia/ reperfusion injury in human kidney transplantation: an immunohistochemical analysis of changes after reperfusion. Am J Pathol. 1998; 153(2):557-566.

12. Gokhan M, Hamdi K, Alp D, Gulnaz A, Munire T, Bilgin N, et al. Evaluation of renal transplant recipients of paired cadaveric kidneys. Transplant Proc. 1999; 31(8):3119-3120.

13. Kyllönen L, Salmela K. Transplantation of both kidneys from 408 donors; comparison of results. Transpl Int. 2000; 13 Suppl 1:S95-98

14. Wilhelm S, Wunderlich H, Zermann D, Werner W, Schubert J. Different graft outcome of paired kidneys from same donorswhy? Transplant Proc. 2000; 32(4):769-70.

15. Halloran PF, Aprile MA, Farewell V, Ludwin D, Smith EK, Tsai SY, et al. Early function as the principal correlate of graft survival. A multivariate analysis of 200 cadaveric renal transplants treated with a protocol incorporating antilymphocyte globulin and cyclosporine. Transplantation. 1988; 46(2):223-228.

16. McLaren AJ, Jassem W, Gray DW, Fuggle SV, Welsh KI, Morris PJ. Delayed graft function: risk factors and the relative effects of early function and acute rejection on long-term survival in cadaveric renal transplantation. Clin Transplant. 1999; 13(3): 266-272.

17. Cosio FG, Alamir A, Yim S, Pesavento TE, Falkenhain ME, Henry ML, et al. Patient survival after renal transplantation: I. The impact of dialysis pre-transplant. Kidney Int. 1998; 53(3): 767-772.

Correspondencia autor: Dr. Juan C. Regueiro López Servicio de Urología

Hospital Regional Universitario Reina Sofía Avda. Menéndez Pidal, 1 - 14004 Córdoba

Tel.: 957010001

E-mail autor: jcregue0@ono.com

Información artículo: Original - Trasplante renal

Trabajo recibido: julio 2008

Trabajo aceptado: noviembre 2008 